\title{
SUPPORTING INFORMATION: Increase of Polymerization Yield on Titania by Surface Reduction
}

Mikel Abadia, ${ }^{\dagger}$ Guillaume Vasseur, ${ }^{\ddagger \dagger \dagger}$ Marek Kolmer,,$₫$ Lukasz Zajac, ${ }^{\Uparrow}$ Alberto Verdini, ${ }^{\prime}$ J. Enrique Ortega, ${ }^{\dagger, \ddagger \perp}$ Luca Floreano, ${ }^{*, \|}$ Celia Rogero, ${ }^{*, \dagger, \neq}$ and Jens Brede $^{*, \dagger}$

$\dagger$ Materials Physics Center CFM-MPC (CSIC-UPV/EHU), 20018 San Sebastian, Spain $\ddagger$ Donostia International Physics Center DIPC, 20018 San Sebastian, Spain \Center for Nanometer-Scale Science and Advanced Materials, NANOSAM, Faculty of Physics, Astronomy and Applied Computer Science, Jagiellonian University, 30348 Krakow, Poland

$\S$ Preset address: Center for Nanophase Materials Sciences, Oak Ridge National Laboratory, Oak Ridge, Tennessee 37831, USA

||CNR-IOM, Laboratorio Nazionale TASC, 34149 Triestre, Italy $\perp$ Departamento Fisica Aplicada I (UPV/EHU), 20018 San Sebastian, Spain E-mail: floreano@iom.cnr.it; celia.rogero@csic.es; dr.jens.brede@gmail.com 


\section{Angle resolved photoemission spectroscopy maps of the polymer}

\section{phases}

$\mathrm{hr}-\mathrm{TiO}_{2}(\mathbf{1 1 0})$

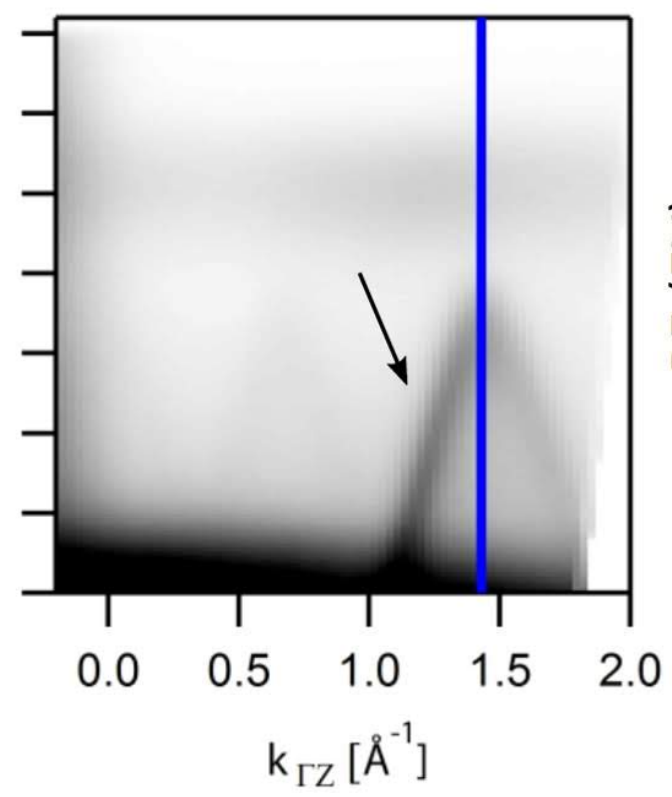

ns- $\mathrm{TiO}_{2}(110)$

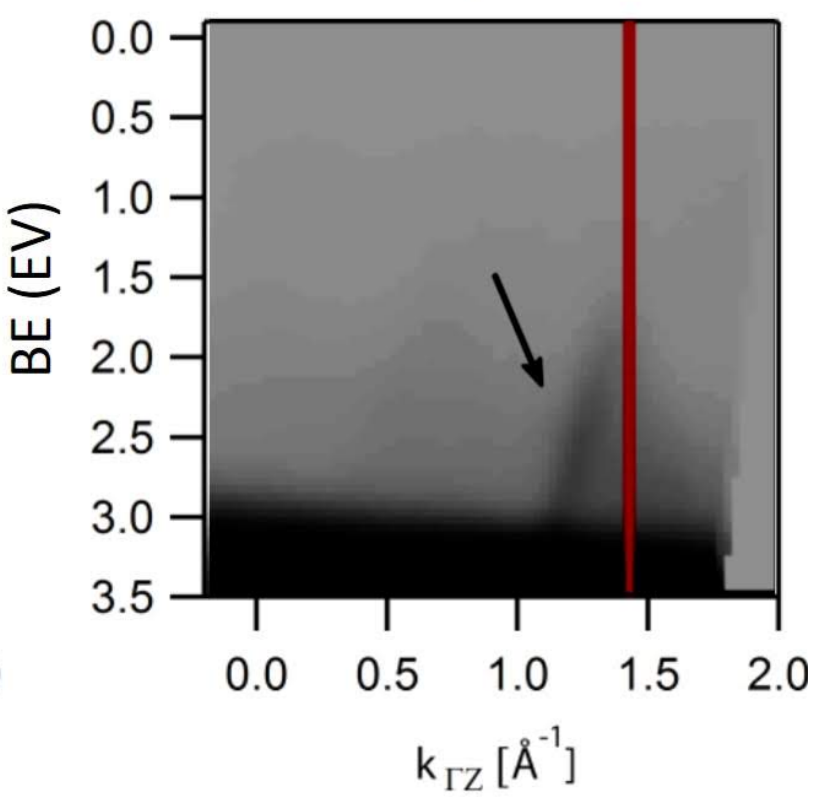

Figure 1: Experimental raw ARPES intensity maps acquired on the highly reduced and nearly-stoichiometric TiO2(110) surfaces on the polymer phases. In both cases a highly dispersive band associated to a well ordeder and long $\pi$-conjugated system is observed. The band measured on the $n s-\mathrm{TiO}_{2}(110)$ is very faint but rather similar to the PPP band obtained in the $h r-\mathrm{TiO}_{2}(110)$. In both cases, the band apex is close $1.45 \AA^{-1}$ and at a maximum energy of around $-2.06 \mathrm{eV}$. The lower integrated intensity of the band measured on the $n s-\mathrm{TiO}_{2}(110)$ means that the amount of polymers from which the signal is originated is considerably lower. 
Difference spectra to highlight molecular orbitals

\section{$\mathrm{hr}^{-\mathrm{TiO}_{2}}$ (110)}

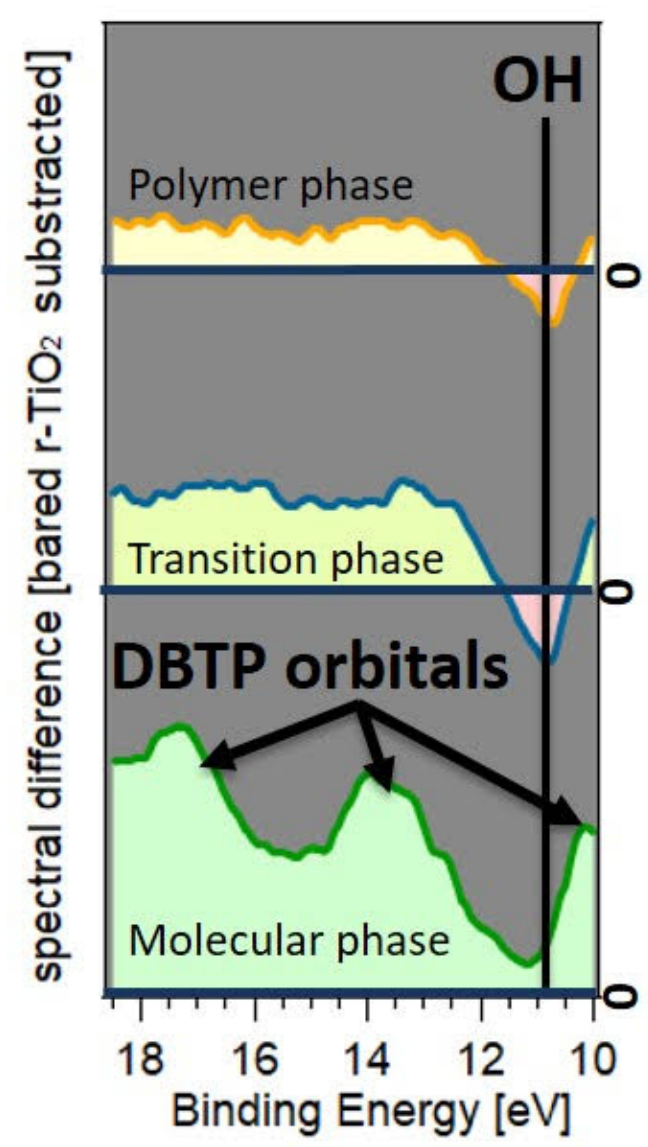

ns- $\mathrm{TiO}_{2}(110)$

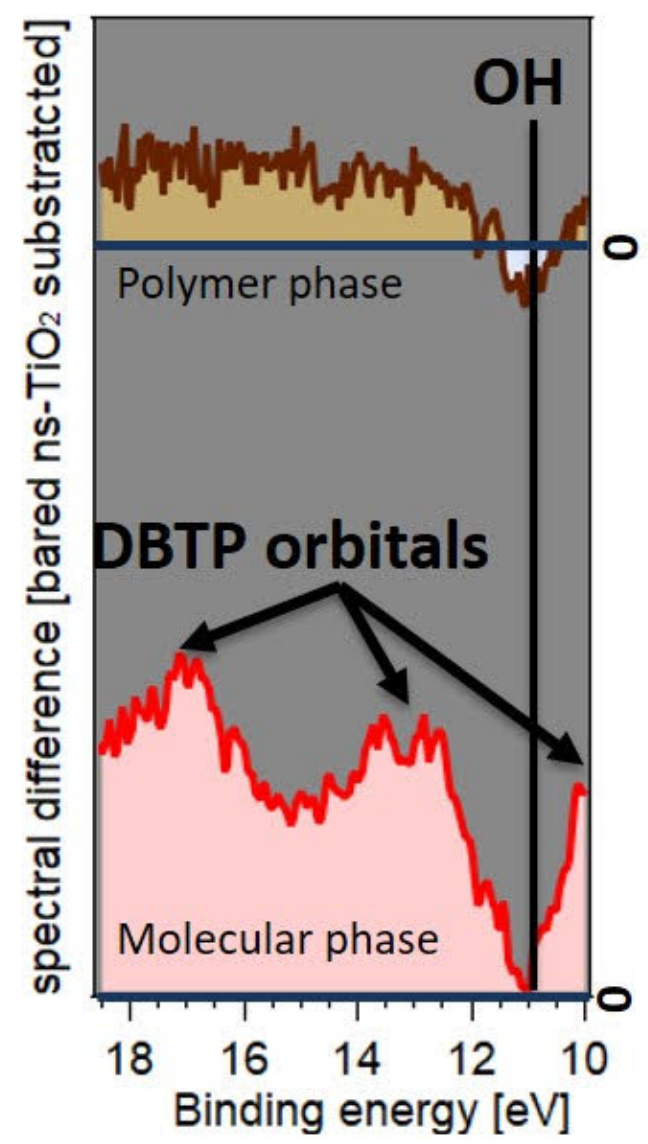

Figure 2: Difference spectra - clean surface emission was subtracted from the supramolecular, transition (only for $h r-\mathrm{TiO}_{2}(110)$ ) and polymer phases - to highlight the transition from sharp molecular features to a flat density of states, in-line with the observed transition from molecular orbitals to dispersing oligomer $\pi$-bands. 